\title{
Distinction des prostatites aiguës récidivantes et chroniques
}

\author{
Jean-Michel BENOIT \\ Service Urologie-Andrologie, CHU Toulouse
}

\begin{abstract}
RESUME
L'E.A.U. (European Association of Urology) a publié en 2001 des recommandations pour la prise en charge des infections du tractus urinaire. Dans le chapitre concernant les prostatites, l'E.A.U. propose notamment de distinguer prostatites aiguës et chroniques en fonction de la durée des symptômes : le diagnostic de prostatite chronique est posé lorsque les symptômes urinaires durent depuis au moins trois mois. Les prostatites aiguës qui se reproduisent (récidivantes donc) ne sont ainsi pas envisagées, alors que leur existence paraît cliniquement très probable, et que la distinction des formes récidivantes et chroniques peut être difficile lors de récidives rapprochées.

L'utilité de la distinction de ces deux formes de prostatite est discutée ici à travers trois questions : est-elle justifiée (existe-t-il des données incontestables dans la littérature) ? A-t-elle des conséquences théoriques et pratiques ? Est-elle réalisable, particulièrement en "pratique courante " ? Ce dernier point est argumenté à l'aide de données personnelles, relevées en pratique de ville. Finalement, il semble justifié de distinguer prostatites aiguës récidivantes et chroniques, mais cette distinction peut être difficile. Elle bute en particulier sur un point : l'affirmation de la désinfection prostatique.
\end{abstract}

Mots clés : prostatites aiguës, prostatites récidivantes, prostatites chroniques, désinfection prostatique

\section{INTRODUCTION}

L'E.A.U. (European Association of Urology) a publié en 2001 des recommandations pour la prise en charge des infections du tractus urinaire [3]. Dans le chapitre concernant les prostatites, l'E.A.U. propose de distinguer prostatites (présence d'une infection) et syndrome douloureux pelvien chronique (même symptomatologie mais absence d'infection démontrée). Le mot de prostatite est donc réservé aux infections aiguës (classées 1 ), chroniques (classées II), et aux prostatites inflammatoires asymptomatiques, ou histologiques (classées IV), ces dernières étant surtout des découvertes de biopsie.

Les prostatites de type I et II, seules formes discutées ici, sont distinguées par l'E.A.U. en fonction de la durée de la symptomatologie. Le diagnostic de prostatite chronique est porté en présence d'une symptomatologie persistant depuis au moins trois mois.

Les prostatites aiguës qui se reproduisent (prostatites récidivantes donc) ne sont pas envisagées dans ces recommandations. Leur existence paraît pourtant cliniquement très probable. Et il peut même arriver, particulièrement en " pratique courante ", qu'elles soient difficile à distinguer des prostatites chroniques, surtout lorsque les récidives d'infections aiguës se rapprochent.

L'utilité de la distinction prostatites récidivantes/prostatites chroniques est discutée en posant trois questions : cette distinction est-elle justifiée (i.e. existe-t-il des données fiables sur ce point dans la littérature) ? Peut-elle avoir des conséquences notables, théoriques et pratiques ? Est-elle possible, particulièrement en pratique extra-hospitalière. Ce dernier point est argumenté en s'aidant de données personnelles, relevées en « pratique de ville».

\section{Correspondance :}

Dr Jean-Michel Benoit - Service d'Urologie-Andrologie (Pr. Plante), Hôpital de Rangueil, TSA 30030, 31059 Toulouse cedex 9. France - Tel 05.61.32.27.31 - Fax 05.61.32.22.85 - Email vermande.f@chu-toulouse.fr 


\section{DONNEES DE LA LITTERATURE}

Les études longitudinales de prostatites clairement documentées sont très rares. La re-lecture des travaux de l'èquipe de Stanford (Stamey puis Shortliffe) fournit des données d'autant plus précises qu'elle utilise la méthode de référence, le test de Meares-Stamey, mis au point par cette même équipe.

Pour mémoire le test de Meares-Stamey [2] consiste à obtenir, lors d'un épisode de prostatite, quatre échantillons : quelques millilitres du premier jet d'urine (VB1), du milieu de jet (VB2), quelques gouttes de sécrétions prostatiques obtenues par massage de la glande (EPS) et les urines obtenues après massage prostatique (VB3). Le nombre de leucocytes et de germes par millilitre est demandé au laboratoire pour chaque fraction recueillie. Lorsqu'on ne peut obtenir d'EPS, une spermoculture peut fournir des indications utiles $[2,10]$. La comparaison des germes et leucocytes par millilitre des différentes fractions permet d'affirmer l'infection urinaire d'une part, et la localisation prostatique d'autre part.

Il est difficile de résumer le gros chapitre du livre de Stamey [9] consacré aux infections prostatiques, et le long article de Shortliffe [8] sur le même sujet. II est possible d'en extraire plusieurs données importantes.

Stamey [9] décrit 13 cas de prostatites, dont 5 sont détaillés lors de suivis de 2 à 12 ans :

- dans deux cas il n'obtient jamais de disparition des germes lors des tests urinaires (dans un cas le germe est un Entérobacter, dans l'autre un Pyocyanique);

- dans trois cas les germes disparaissent après traitement, avant ré-apparition d'un germe de séro-type différent, à savoir : E. Coli 06 puis E.Coli 075 (intervalle entre les infections : 6 mois) ; E. Coli 011 puis E. Coli 075 (intervalle : 2,5 mois) ; E. Coli 04 puis E. Coli SA (intervalle : 5,5 ans).

Shortliffe [8] détaille l'histoire de 13 hommes atteints de prostatite, avec des suivis de 2 à 6 ans :

- un patient présente 4 prostatites en 6 ans, avec des germes toujours différents : E. Coli 01, 075, 016, NT. Deux infections se succèdent en 5,5 mois. Chaque infection est traitée et stérilisée ;

- dans 8 cas, la stérilisation est obtenue par le traitement antibiotique, y compris en cas de nouvelle infection ultérieure ;

- dans 4 cas, la stérilisation n'est jamais atteinte. Le germe isolé est $\mathrm{E}$. Coli dans 2 cas, Entérobacter dans 1 cas, Pyocyanique et E. Coli dans 1 cas.

Quatre points importants se dégagent de ces travaux. D'abord l'existence d'infections successives à germes différents authentifie la notion de prostatites récidivantes sur le plan bactériologique. Ensuite ces infections récidivantes peuvent se reproduire à intervalles aussi courts que 2,5 mois. En troisième lieu, les prostatites avec persistance du même germe, donc bactériologiquement chroniques, sont moins fréquentes que les prostatites récidivantes. Enfin les germes mis en évidence sont différents dans ces deux formes de prostatite : E. Coli est prédominant dans les formes récidivantes, Entérobacter et Pyocyanique dans les formes chroniques.

\section{CONSEQUENCES DE LA DISTINCTION RECIDIVANT/CHRONIQUE}

En dehors des cas de traitement manifestement inadapté (produit choisi et/ou durée de traitement incorrects) et des cas évidents (deux prostatites séparées par un intervalle de 5,5 ans, cf. supra), la reconnaissance des formes de prostatite récidivante et leur différenciation des cas de prostatite chronique, a des conséquences théoriques et pratiques.

Les prostatites récidivantes impliquent la notion de nouvelle infection (récidive), donc de maîtrise antibiotique de l'infection précédente.

Elles s'inscrivent dans le cadre d'un succès thérapeutique médical. Schématiquement, ce contexte amène à s'interroger plutôt sur l'existence d'un " défaut biologique » (adhérence ? colonisation urétrale ? défaut de zinc (" antibiotique naturel ") persistant ou passager (saignement intraprostatique ? etc...) et à tenter de le corriger, si celà est possible. De façon plus pratique, il amène aussi à rechercher un facteur favorisant ou une cause déclenchante (circonstances produisant une " congestion prostatique », des " micro-traumatismes du périnée ", tels voyages en auto, pratique du vélo, etc...).

Les prostatites chroniques impliquent une persistance bactérienne, condition importante pour affirmer la chronicité, et témoignent schématiquement d'un échec du traitement antibiotique. Elles s'inscrivent dans le cadre d'un échec thérapeutique médical, en dehors bien sûr des cas de traitement inadapté. Elles amènent à évoquer un " foyer de persistance des germes ", à le rechercher et à le traiter si possible : calculs prostatiques, nodule d'épididymite chronique, vésiculite en sont des exemples.

Les conséquences pratiques de la distinction prostatites récidivantes/chroniques sont de deux ordres. D'abord les traitements en sont différents : antibiotiques plus « puissants " et pour une durée plus longue sont indiqués en cas de prostatite chronique [3]. Ces antibiotiques ne sont pas dépourvus d'effets secondaires, et doivent dans certains cas être manipulés avec précaution [3].

Ensuite cette distinction soulève plusieurs questions. Doiton traiter une prostatite dont les récidives se rapprochent comme autant de prostatites aiguës ou est-il licite de prolonger les traitements ? Peut-on envisager des traitements filés, comme cela se pratique dans le cas des cystites ? Peut-on imaginer de traiter ponctuellement une sujet chez lequel une cause déclenchante paraît identifiée ? A l'inverse doit-on intervenir chirurgicalement plus souvent : par exemple en présence de calculs prostatiques $[9,10]$ ? 


\section{LA DISTINCTION RECIDIVANT / CHRONIQUE EN PRATIQUE}

En dehors des cas évidents (épisodes de prostatite séparés par un intervalle long) ou des traitements manifestement inadaptés, la distinction prostatites aiguës récidivantes / prostatites chroniques peut poser de réelles difficultés, particulièrement en pratique extra-hospitalière. Les données personnelles qui suivent [1] sont les principaux renseignements recueillis lors d'une première consultation de patients porteurs de prostatite vus dans la station thermale de La Preste.

\section{Dossiers et classement}

Soixante seize dossiers de patients présentant une symptomatologie de prostatite, correctement explorés (urographie intra-veineuse et urétro-cystographie mictionnelle, ou abdomen sans préparation et échographie du haut et du bas appareil urinaire, excluant une pathologie autre que prostatique), sont présentés. Ils comportent en outre un bilan sanguin minimal (glycémie, créatinine, vitesse de sédimentation, numération-formule sanguine) qui est normal. La documentation bactériologique de ces dossiers comporte les examens cyto-bactériologiques disponibles (ECBU), qui sont soit des examens urinaires $2^{e ̀ m e}$ jet, soit des examens 1 er et 2ème jets, soit des spermocultures, soit 1er 2ème jets et spermoculture. Au moins trois examens clairement démonstratifs d'infection sont exigés. Les examens de contrôle sont considérés comme stériles en présence d'un compte de leucocytes $/ \mathrm{ml}$ normal et d'un compte de germes nul (ou pour les Staphylocoques et Streptocoques inférieur à $10.000 / \mathrm{ml}$, Entérocoques exclus).

La distinction entre prostatites récidivantes (PAR) et chroniques (PCB) s'appuie sur la clinique (repérage entre des poussées caractérisées d'une période asymptomatique d'au moins un mois avec un contrôle urinaire stérile) et la bactériologie disponible (étude des examens urinaires ou spermocultures et recherche d'une possible persistance bactérienne : germe en cause et comparaison des antibiogrammes).

Dans ces conditions 53 patients $(69,7 \%)$ sont classés dans la catégorie " prostatites récidivantes », 23 " prostatites chroniques ». La classification est difficile dans 4 cas $(5,2 \%)$.

\section{Comparaison des deux groupes}

\section{a) Données d'interrogatoire :}

1. Les 53 PAR ont présenté durant l'année précédant la consultation 0 à 6 crises de prostatite caractérisées quant aux symptômes urinaires, mais inconstamment fébriles. Deux patients $(3,8 \%)$ sous antibiothérapie prolongée n'ont pas eu de crise, $8(15,1 \%)$ ont eu une crise, $14(26,4 \%)$ deux crises, $12(22,6 \%)$ trois crises, et $17(32,1 \%)$ quatre crises ou plus. Ce type de décompte n'est pas réalisable chez les patients classés $\mathrm{PCB}$.

2. Des douleurs urinaires entre les poussées aiguës sont mentionnées : $19 / 53(35,8 \%)$ pour les PAR versus $14 / 23$ $(60,1 \%)$ chez les PCB ( $p=0,049$; test exact de Fisher).
3. Antécédents documentés de maladie sexuellement transmissible : $4 / 53(7,5 \%)$ pour les PAR versus $6 / 23$ $(26,1 \%)$ pour les PCB $(p=0,058)$.

\section{b) Etat prostatique :}

En tenant compte de toutes les données disponibles, radiologiques, échographiques, de toucher rectal, et en négligeant les petites augmentations de volume :

1. La prostate est normale pour $32 / 53(60,4 \%)$ des PAR versus $3 / 23(13,0 \%)$ des PCB $(p<0,001)$.

2. Une injection intra-prostatique de produit de contraste est observée chez : $6 / 53(11,3 \%)$ des PAR versus $12 / 23$ $(52,2 \%)$ des PCB $(p<0,001)$.

3. Des calculs ou calculins radio-opaques prostatiques sont présents chez : $3 / 53(5,7 \%)$ des PAR versus $10 / 23$ $(43,5 \%)$ des PCB $(p<0,001)$.

\section{c) Données bactériologiques :}

Durant l'année précédant la consultation, on dispose de 103 ECBU (ou spermocultures) pour les 53 PAR et de 65 ECBU/spermocultures pour les $23 \mathrm{PCB}$. Les germes de culture urinaire ou de spermoculture qui ont été mis en évidence sont rapportés au Tableau 1.

\section{Immunoglobulines séminales totales}

Les immunoglobulines séminales $A$ et $G$ ( $\lg A$ et $\lg G$ ) augmentent au cours d'une infection prostatique et se normalisent à distance de celle-ci $[4,8,9]$. Leur dosage peut donc permettre de confirmer la désinfection prostatique. Si le dosage des immunoglobulines spécifiques n'est pas accessible en pratique extra-hospitalière, le dosage des IgA et IgG séminales totales (spécifiques + non-spécifiques) est réalisable " en ville » en recourant à un laboratoire spécialisé. II a été appliqué à 7 témoins pour étalonner la méthode, et 20 sujets de la série ci-dessus soit : 3 sujets présentant une prostatite en cours ou récente, et 17 sujets qui, lors du suivi, paraissent désinfectés depuis un an au moins, selon les critères cliniques et bactériologiques décrits précédemment. Le dosage fournit les résultats rapportés au Tableau 2, conformes à ceux de la littérature.

Tableau 1 : Type et fréquence des germes retrouvés dans les ECBU ou spermocultures durant l'année précédant la consultation.

\begin{tabular}{lccc}
\hline & PAR & PCB & Test de Fisher \\
\hline 1. Escherichia Coli & $75 / 103$ & $37 / 65$ & $\mathrm{p}=0,044$ \\
& $(72,8 \%)$ & $(56,9 \%)$ & \\
2. Entérocoque & $6 / 103$ & $9 / 65$ & non significatif \\
& $(5,8 \%)$ & $(13,8 \%)$ & \\
$\begin{array}{l}\text { 3. Klebsielle ou } \\
\text { Moraxelle }\end{array}$ & $2 / 103$ & $0 / 65$ & non significatif \\
$\begin{array}{l}\text { 4. Serratia ou } \\
\begin{array}{l}\text { Pyocyanique } \\
\text { Pyon }\end{array}\end{array}$ & $0 / 103$ & $9 / 65$ & $\mathrm{p}<0,001$ \\
& & $(13,8 \%)$ & \\
\hline
\end{tabular}


Tableau 2 : Résultats du dosage des lgA et lgG séminales totales.

\begin{tabular}{lccc}
\hline Populations & & IgA & IgG \\
\hline 7 sujets témoins & & $<50 \mathrm{mg} / \mathrm{l}$ & $<200 \mathrm{mg} / \mathrm{l}$ \\
3 cas de prostatite & cas 1 & $80 \mathrm{mg} / \mathrm{l}$ & $300 \mathrm{mg} / \mathrm{l}$ \\
& cas 2 & $100 \mathrm{mg} / \mathrm{l}$ & $220 \mathrm{mg} / \mathrm{l}$ \\
& cas 3 & $220 \mathrm{mg} / /$ & $510 \mathrm{mg} / \mathrm{l}$ \\
17 sujets désinfectés & & \\
& 16 cas & $<50 \mathrm{mg} / /$ & $<200 \mathrm{mg} / \mathrm{l}$ \\
& 1 cas & $200 \mathrm{mg} / 1$ & $150 \mathrm{mg} / \mathrm{l}$ \\
\hline
\end{tabular}

\section{DISCUSSION}

Il existe des données bactériologiques fiables dans la littérature, qui confirment les données cliniques, et leur fournissent un substrat bactériologique solide : cela autorise à individualiser le tableau de prostatite récidivante. Cette forme d'infection prostatique soulève des problèmes différents de ceux de la prostatite chronique. Schématiquement les questions se posent de façon théorique dès le deuxième épisode de prostatite (quelle peut être la cause de cette récidive infectieuse ?). Elles se posent de façon pratique (faut-il modifier le traitement ?) dès que les prostatites se rapprochent.

La therrapeutique est assez clairement fixée en cas de prostatite chronique $[3,5,6]$, et à notre connaissance non documentée pour les prostatites récidivantes. Cela ne concerne pas les prostatites récidivant après des intervalles longs, et qui semblent logiquement pouvoir être traitées comme des prostatites aiguës, mais les prostatites se reproduisant à intervalles rapprochés, ces intervalles n'étant d'ailleurs pas définis. La série de prostatites présentée ci-dessus montre en outre que la distinction des deux tableaux devient difficile en présence de prostatites pluriannuelles. Un élément important de cette difficulté est la fréquence d'apparition d'E. Coli qui rend malaisée, avec des données bactériologiques courantes, la différenciation des récidives et des rechutes infectieuses.

Lorsqu'on ne dispose pour distinguer les deux tableaux que des données usuelles, cliniques et bactériologiques, la classification est hésitante dans $5 \%$ des cas environ. La persistance de douleurs urinaires entre crises mieux caractérisées s'avère un critère qui discrimine tout juste les formes récidivantes et chroniques, alors que cette classification se revvèle par ailleurs assez satisfaisante. Elle est très bien corrélée à l'état prostatique, elle confirme la liaison avec les antécédents de maladie sexuellement transmissibles, et la notion bactériologique de plus grande fréquence d'E.Coli dans les formes récidivantes. Cependant il ne s'agit là que de liaisons statistiques, donc globales. La douleur périnéale, symptôme très spécifique de la prostatite [7] n'apporte ici aucun renseignement supplémentaire.

Une difficulté majeure en pratique extra-hospitalière reste l'affirmation de la désinfection prostatique. Le test de Mea-
res-Stamey est d'application très difficile dans ce contexte, bien qu'il constitue le meilleur test connu de désinfection, en cas de stérilité de la fraction EPS. II est donc fréquent de devoir se contenter de la spermoculture [10], laquelle n'est vraiment fiable que si elle est parfaitement stérile, et encore plus fréquemment de la bactériologie du $2^{\text {ème }}$ jet urinaire, qui, même stérile, laisse toujours planer un doute. L'argument temps, lors de poussées de prostatites espacées, est de bonne valeur pour confirmer la désinfection. Un temps minimum n'est cependant pas clairement défini.

Le dosage des immuno-globulines séminales $A$ et $G$ fournit un marqueur connu et documenté de l'état infectieux de la prostate $[4,8,9]$. La corrélation entre IgA et infection à partir des données rapportées ci-dessus est de $0,84(p=$ $0,0035)$. Pour les IgG, cette corrélation est de $1(p=$ 0,0009 ). Elles ont cependant le grave inconvénient de se normaliser très lentement, en 12 à 18 mois. Cela se vérifie pour les mêmes données : l'un des 17 sujets présumés désinfectés depuis au moins un an présente un taux d'lgG normalisé, alors que les IgA sont encore élevées. De plus la survenue rapprochée de deux prostatites aboutit la deuxième fois à une nouvelle augmentation d'immunoglobulines à partir d'un taux déjà élevé, retardant encore la normalisation de celles-ci, qui peut alors dépasser deux ans. Dès que les épisodes de prostatites deviennent pluriannuels, cette méthode devient donc inapplicable.

\section{CONCLUSIONS}

La distinction des prostatites récidivantes et chroniques paraît une proposition raisonnable particulièrement en cas de récidives assez rapprochées et / ou de douleurs urinaires persistantes amenant à s'interroger sur le type d'évolution de l'infection. La prolongation des douleurs urinaires, qui serait selon la classification E.A.U. le critère distinguant prostatites aiguës et chroniques, n'est pas un caractère de différenciation simple dans la série rapportée plus haut. Par ailleurs, la distinction de formes aiguës et chroniques sans référence bactériologique, pose question.

Si cette distinction est admise, les conséquences en sont de deux ordres. Sur le plan clinique la prostatite récidivante pourrait être placée entre la prostatite aiguë et la prostatite chronique. II paraît logique de traiter comme deux épisodes aigus deux prostatites séparées par un intervalle de temps long. Mais lorsque les récidives de prostatites sont par exemple pluriannuelles, un certain nombre de questions émergent, qui n'ont pas à ce jour de réponse claire. Faut-il étudier des modalités particulières de traitement ? Quel rythme d'infection retenir pour envisager de tels traitements ? Quelle est la pathogénie de telles infections? Enfin, quelle attitude adopter pratiquement, surtout en exercice extra-hospitalier?

Pour ce dernier point, il apparaît qu'obtenir un échantillon de sperme et des urines de $1^{\text {er }}$ et $2^{\text {ème }}$ jet est la moins difficile des options proposées par le test de 
Meares-Stamey. Mais on aura compris que si la documentation d'une infection prostatique n'est pas obligatoirement compliquée, la certitude de la désinfection reste un point crucial qui pose très souvent de réelles difficultés.

\section{REFERENCES}

1. BENOIT J-M., JEANJEAN P. : données non publiées.

2. BOCCON-GIBOD L., BAERT L. : Les prostatites. In : Khoury S ed. Urologie, pathologie infectieuse et parasitaire. Paris, Masson, 1985 : 278-284.

3. E.A.U. : Guidelines on Urinary and Male Genital Tract Infections. Arnhem, Drukkerij Gelderland, 2001 : 49-56.

4. FOWLER J.E. Jr: Bacteriuria and associated infections of the reproductive system in men. In : Fowler J.E. Jr : Urinary Tract Infection and Inflammation, 1989, 92-123.

5. JOHNSON J.R. : Treatment and prevention of urinary tract infections. In : Mobley H.L., Warren J.W. eds. Urinary Tract Infections. Molecular Pathogenesis and Clinical Management. Washington, ASM Press, 1996 : 95-118.

6. KUNIN C.M. : Infections in males. In : Kunin C.M. ed. Detection, Prevention and Management of Urinary Tract Infections. Philadelphia, Lea \& Febiger, 1987 : 356-361.

7. LITWIN M.S., McNAUGHTON-COLLINS M., FOWLER F.J. Jr, et al : The National Institutes of Health Chronic Prostatitis Symptom Index : Development and validation of a new outcome measure. J. Urol., $1999: 369-375$.

8. SHORTLIFFE L.M., WEHNER N. : The characterization of bacterial and nonbacterial prostatitis by prostatic immunoglobulins. Medicine, 1986 : 399-414.

9. STAMEY T.A. : Urinary infections in males. In : Stamey T.A. ed. Pathogenesis and Treatment of Urinary Tract Infections. Baltimore, Williams \& Wilkins, $1980: 342-429$.

10.WEIDNER W., LUDWIG M., SCHIEFER H.G. : Chronic bacterial prostatitis - A clinical reevaluation of old woes. In : Bergan T. ed. Urinary Tract Infections. Bale, Karger, 1997 : 60-66.

Communication au $X X^{\circ}$ Congrès de la Société d'Andrologie de Langue Française, Orléans, 11-13 décembre 2003.

Travail réalisé pour le Comité Infectiologie de l'AFU

Manuscrit reçu : décembre 2003 ; accepté : janvier 2004.

\section{ABSTRACT}

Distinction between recurrent and chronic prostatitis

Jean-Michel BENOIT

The E.A.U. (European Association of Urology) published its Guidelines on Urinary and Male Genital Tract Infections in 2001. In the chapter devoted to prostatitis, epididymitis and orchitis, the E.A.U suggests a classification distinguishing prostatitis (usual clinical picture and demonstrated infection) from chronic pelvic pain syndrome (same clinical picture without demonstrable infection). Prostatitis is divided into three categories : acute bacterial prostatitis (type I), chronic bacterial prostatitis (type II) and asymptomatic inflammatory prostatitis (histological prostatitis, type IV). Type I and II prostatitis are considered here. The E.A.U. guidelines do not mention recurrent prostatitis. The authors discuss whether or not recurrent prostatitis should be distinguished from chronic prostatitis by raising three questions: does the literature provide precise data in favour of this distinction? Does this theoretical distinction have any practical consequences? is this distinction feasible, especially in general practice?

The Stanford research team (Stamey and Shortliffe) has provided documented bacteriological data demonstrating recurrence of prostatitis with different bacteria in some cases and persistence of the same pathogen in other cases. The main consequence of these two situations concern treatment (which antibiotics? for how long?). On the basis of personal unpublished data, the authors discuss the feasibility of this distinction in general practice. They show that, in the case of several recurrences of prostatitis per year, it may be difficult to distinguish recurrent prostatitis from chronic prostatitis. They also show that the duration of symptoms is not a sufficiently discriminant factor and that bacteriological findings should be considered.

In conclusion, recurrent prostatitis is a particular disease which should be distinguished from chronic prostatitis. The main consequence of this distinction concerns several unresolved questions about the therapy of recurrent prostatitis. In general practice, the distinction may be difficult when only routine bacteriological tests are available. The use of Meares and Stamey's four glass technique is unusual in this setting, making it difficult to confirm prostatic disinfection.

Key-Words: acute prostatitis, recurrent prostatitis, chronic prostatitis, prostatic disinfection. 